\title{
Rupture of a pseudoaneurysm in brachial artery after hemodialysis therapy: rare but serious complication
}

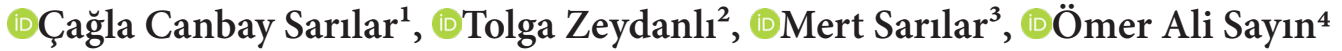 \\ ${ }^{1}$ Ardahan State Hospital Department of Cardiovascular Surgery, Ardahan, Turkey \\ ${ }^{2}$ Ardahan State Hospital Department of Radiology Ardahan, Turkey \\ ${ }^{3}$ Ardahan State Hospital Department of Cardiology Ardahan, Turkey \\ ${ }^{4}$ İstanbul University Faculty of Medicine, Department of Cardiovascular Surgery, İstanbul, Turkey
}

Cite this article as: Canbay Sarılar Ç, Zeydanlı T, Sarılar M, Sayın ÖA. Rupture of a pseudoaneurysm in brachial artery after hemodialysis therapy: rare but serious complication. Anatolian Curr Med J 2022; 4(1); 123-125.

\begin{abstract}
Pseudoaneurysm (PSA) is a common complication that may occur on the AV fistula line. However, the 5 development of the PSA on a wrong cannulated brachial artery is a rarely seen and catastrophic 6 complication as occured in this case. Rupture is one of the serious complication of PSA. In our case 64 years old male with left snuff-box arteriovenous fistula (AVF) refer to emergency service with pain, swelling, tension on left arm after hemodialysis session. Doppler ultrasound confirmed brachial artery ruptured PSA active bleeding in to the approximately $4 \mathrm{~cm}$ diameter hematoma. The patient was transferred to the operating room and underwent to surgery for ruptured PSA repair. Four weeks after surgery, the arteriovenous fistula was used as an access for hemodialysis. Left hand 1st and 2nd digits distal phalanx flexion was limited Clinical suspicion, physical examination, and Doppler ultrasound are essential for early diagnosis and for optimal treatment. Doppler ultrasound can detect the lesion accurately. In patients with arteriovenous fistula during hemodialysis it should be considered pseudoaneurysm, rupture and neuropathy may occur. These are rare but serious complications so suspicion, clinical examination and doppler ultrasound are very important for early diagnosis and treatment.
\end{abstract}

Keywords: Hemodialysis, brachial artery pseudoaneurysm, pseudoaneurysm rupture

\section{INTRODUCTION}

Pseudoaneurysms or false aneurysms are pulsatil masses which is encircled by a fibrous capsule and which has anomalous connection between a ruptured vessel and the soft tissue (1). Causes of PSAs are Ehler Danlos Syndrome, Kawasaki's disease, mycotic aneurysm, trauma and iatrogenic (2). Iatrogenic PSAs rate has increased due to increasing endovascular arterial interventions such as endovascular diagnostic and therapeutic procedures and in advertent puncture of vascular access in hemodialysis patients (3). Peripheric artery PSA are rare in upper extremity than lower extremity (4). Brachial artery PSA early diagnosis and surgical treatment are very important they can cause severe complications as upper extremity and finger loss (5). PSA can lead rupture and hemorrhage, neurologic deficit, local infection or ischemia (6).

We report a case of a patient with ruptured PSA of the brachial artery due to unsuccessful venous cannulation after a hemodialysis session.

\section{CASE REPORT}

A 64-year-old male patient was admitted to the emergency department with pain, swelling, and tension on left arm. His medical history was hypertension, coronary bypass surgery 4 years ago, and snuffbox AVF in the left arm had been under hemodialysis for 4 years. After hemodialysis session, the patient had tension, pain in the left arm. Left brachial artery, radial and ulnar artery pulses were palpated and the snuffbox AVF thrill was positive. Left hand $1^{\text {st }}$ and $2^{\text {nd }}$ digits distal phalanx flexion was limited. Doppler ultrasound confirmed active bleeding approximately $4 \mathrm{~cm}$ diameter hematoma which was originated from the brachial artery in left arm. Patient was taken urgently to the operating room. The surgery was performed under general anesthesia. Left brachial artery was surgically exposed. After dissection fibrous capsule and active bleeding was seen in the puncture site of brachial artery. Hemostasis was achieved by 6.0 proline stitch. Postoperative temporary internal jugular vein dialysis catheter was inserted. 


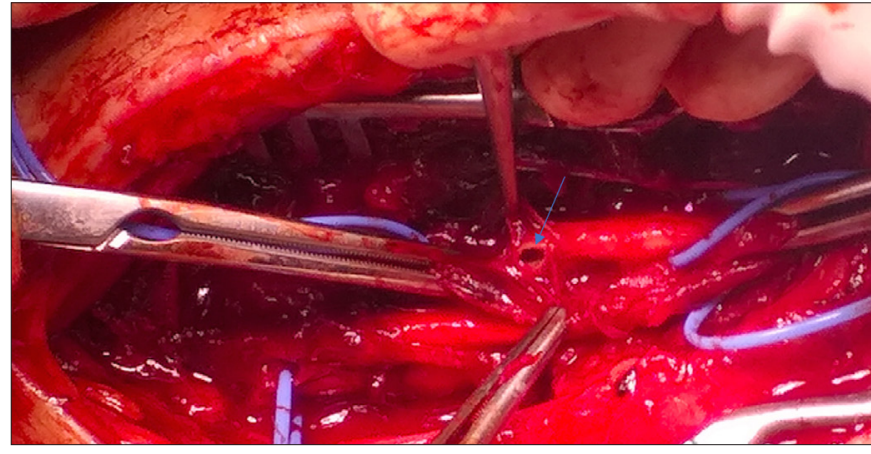

Figure 1. Perioperative ruptured brachial artery PSA neck

Postoperative there were no additional circulatory problems and AVF thrill was positive. The $1^{\text {st }}$ and $2^{\text {nd }}$ digits distal phalanx flexion was limited. Patient had an uneventful recovery and he discharged postoperative $5^{\text {th }}$ day without any deficit in the left arm.

After operation patient received hemodialysis from his temporary hemodialysis catheter and after 4 weeks AVF was used again. Postoperative control ultrasonography confirmed the integrity of the brachial artery.

\section{DISCUSSION}

Upper extremity PSAs are rarer than lower extremity. The etiology of PSAs are congenital diseases such as EhlersDanlos syndrome, polyarteritis nodosa, Behçet disease, Kawasaki disease, osteochondromas or to iatrogenic complications such as diagnostic, therapeutic and accidental punctures or drug abuse $(2,6)$.

Brachial artery PSAs are mainly due to traumas, cannulations and blood gas samplings $(3,7)$.

Patients with arteriovenous fistula has high risk for developing iatrogenic PSAs due to repeated cannulation and heparinization. After traumatic penetration of the vessel hemorrhage and extravasation can occur. The brachial artery PSA usually develops slowly. It tooks days to months even years to be detected clinically. Brachial PSA usually presents with erythema, induration and expanding painful pulsatile mass. Sometimes it can be with cyanosis, loss of pulsation, paresthesia, nerve compression (8). There are many reported brachial artery PSA due to hemodialysis venous puncture but there is few cases with ruptured PSA with arteriovenous fistula. In the presence of a vascular complication early diagnosis is very essential for adequate treatment. For early diagnosis, clinical suspicion and being aware of pulsatile mass is important. For diagnosis, many imaging modalities can be used as arterial Doppler sltrasonography, conventional angiography, computed tomography (CT) angiography and magnetic resonance imaging (MRI). Angiography is the gold Standard diagnostic method (9). Doppler ultrasonography is faster, cost-effective and easy accessible imaging method (10). Complications are infection, hemorrhage, distal vascular insufficiency and rupture of the PSA $(3,7,12,13)$.

Treatment of brachial PSAs depends on the location, size and the pathogenesis of the PSAs (11). Surgical methods like ligation, resection and reanastomosis or vein graft interpositioning can be performed. Endovascular methods such as endovascular stent-graft implantations and embolization of sac can be used. US-guided external compression and percutaneous thrombin injection can be used for treatment. US-guided compression can also applied in brachial artery PSA treatment (14). In patients whom taking anticoagulation, long procedural time and patient discomfort are limitations for US-guided compression.

Surgical indications are rapidly expanding PSA, distal ischemia, neuropathy or ischemic soft tissues and skin caused by local pressure, infected PSA, failure of percutaneous treatment (15). Surgical excision and arterial reconstruction is the Standard treatment. Arterial continuity is provided with end-to-end anastomosis or a venous interposition graft $(16,17)$.

When there is infection, limb ischemia, skin ischemia, coexisting large hematomas with compartment syndrome like our case US-guided compression therapy is contraindicated (15).

After hemodialysis session PSA was not suspected at first because there was only small hematoma that we thought the origin was cephalic vein. PSA generally present weeks to months after blunt or penetrating trauma $(3,12)$. In our patient 12 hours after he referred to emergency service with pain, tension and compartment syndrome. Patient with snuff-box AV fistula, brachial artery puncture is unexpectable. It may be related with unexperienced dialysis technician and nurses. Early diagnosis is very essential for planning adequate treatment. We have observed rupture of the brachial artery PSA and neuropathy due to pressure on an adjacent nerve. Despite the successful surgical treatment in the 1 th and 2 nd digits distal phalanx flexion was limited. Third month after the surgery, neuropathy recovered. Forth weeks after the surgery, AV fistulas gets used.

\section{CONCLUSION}

Rupture of brachial artery PSA is very rare after hemodialysis therapy in a patient with AV fistula. Incidence of brachial artery PSA is increasing due to endovascular techniques and arterial cannulations. These complications should be considered by emergency physicians, cardiovascular surgeons, nephrologists and hemodialysis nurses to avoid delayed diagnosis and treatment. Emergent surgical repair is essential for good 
results. Surgery is especially indicated in compartment syndrome with large hematoma.

\section{ETHICAL DECLARATIONS}

Informed Consent: Written informed consent was obtained from all participants who participated in this study.

Referee Evaluation Process: Externally peer-reviewed.

Conflict of Interest Statement: The authors have no conflicts of interest to declare.

Financial Disclosure: The authors declared that this study has received no financial support.

Author Contributions: All of the authors declare that they have all participated in the design, execution, and analysis of the paper, and that they have approved the final version.

\section{REFERENCES}

1. Esteban MP, Otálora SB, Landeras RM, et al. Posttraumatic pseudoaneurysm of the brachial artery and postsurgical retraction of median nerve: description of a case and ultrasonography findings. Emerg Radiol 2007; 13: 269-72.

2. Lee JY, Kim H, Kwon H, Jung S-N. Delayed rupture of a pseudoaneurysm in the brachial artery of a burn reconstruction patient. World J Emerg Surg 2013; 8.

3. Cina G, Rosa MG, Viola G, Tazza L. Arteriel injuries following diagnostic,therapeutic and accidental arterial cannulation in hemodialysis patients. Nephrol Dial Transplant 1997; 12: 144852.

4. Wielenberg A, Borge MA, Demos TC, Lomasney L, Marra G. Traumatic pseudoaneurysm of the brachial artery. Orthopedics 2000; 23: 1250-1322-4.

5. Nugud OO, Hedges AR. Axillary artery pseudoaneurysm. Int J ClinPract2001; 55: 494-9.

6. Kemp K, Radwan R, Shingler G, Davies C. Brachial artery pseudoaneurysm. BMJ Case Rep 2014; pii: bcr2014203924.

7. Lapus TP, Trerotola SO, Savader SJ. Radial artery pseudoaneurysm complicating a brecia-cimino dialysis fistula. Nephron 1996; 72: 673-5.

8. Habermann ET, Cabot WD: Median nerve compression secondary to false aneurysm of the brachial artery. Bull Hosp Joint Dis 1974; 35: 158-61.

9. Ho PK, Weiland AJ, McClinton MA, Wilgis EF: Aneurysms of the upper extremity. J Hand Surg 1987;12: 39-46.

10. Pelaz Esteban M, Beltran de Otalora S, Landeras RM, Gallardo E, Fernandez Echevarria MA, Perez Aguilar D. Posttraumatic pseudoaneurysm of the brachial artery and postsurgical retraction of median nerve: description of a case and ultrasonography findings. Emerg Radiol 2007; 13: 269-72

11. Yetkin U, Gurbuz A. Post-traumatic pseudoaneurysm of the brachial artery and its surgical treatment. Texas Heart Institute journal/from the Texas Heart Institute of St Luke's Episcopal Hospital, Texas Children's Hospital 2003, 30: 293-7.

12. Demircin M, Peker O, Tok M, Özen H. False aneurysm of the brachial artery in an infant following attemtedvenipuncture. Turkish J Pediatr 1996; 38: 389-91.

13. Popovsky MA, McCarty S, Hawkins RE. Pseudoaneurysm of the brachial artery: a rare complication of blood donation. Transfusion 1994;34: 253-4.
14. Kehoe ME. US-guided compression repair of a pseudoaneurysm in the brachial artery. Radiology 1992; 182: 896.

15. Morgan R, Belli AM: Current treatment methods for postcatheterization pseudoaneurysms. J Vasc Intervent Radiol JVIR 2003; 14: 697-710.

16. Siu WT, Yau KK, Cheung HY, et al. Management of brachial artery pseudoaneurysms secondary to drug abuse. Ann Vasc Surg 2005, 19: 657-61.

17. Pages ON, Alicchio F, Keren B, et al. Management of brachial artery aneurisms in infants. Pediatr SurgInt 2008; 24: 509-13. 